\title{
BILATERAL SINGLE-SHOT QUADRATUS LUMBORUM BLOCK FOR POST-OPERATIVE PAIN RELIEF IN A BREASTFEEDING WOMAN
}

Luísa Ferreira ${ }^{1}$, Pedro Cunha ${ }^{1}$, Raquel Oliveira ${ }^{1}$, Carlos Almeida ${ }^{2}$, José Pedro Assunção ${ }^{3}$

1- Anesthesiology residents; 2- Anesthesiologist; 3- Dir. of Anesthesiology Department of

Centro Hospitalar Tondela-Viseu, CHTV

\section{INTRODUCTION}

Post-laparotomy pain is often difficult to control, and intravenous analgesia must be careful in breastfeeding women. Bilateral single-shot quadratus lumborum block (QLB) produces sensitive block from T6-L2 which promotes visceral and somatic pain relief for an extended period, diminishing the need of a continuous technique.

\section{CASE REPORT}

Breastfeeding woman, 39, ASA I, with fever and leukocytosis, PCR 15,99 $\mathrm{mg} / \mathrm{dL}$. Exploratory laparotomy was performed by dehiscence of the uterine anastomosis, 6 days after cesarean section. A general anesthesia was performed, with multimodal analgesia in the intraoperative period. A bilateral QLB was performed with ropivacaine $0.375 \%, 20 \mathrm{ml}$ each side, ultrasound guided at the of end the surgery. The patient reported pain EN 0/10, without requirement of aditional analgesic therapy at the PACU. Paracetamol $1 \mathrm{~g} 3$ tid was added to the analgesic regimen. Patient reported pain EN 2/10 up to $36 \mathrm{~h}$ post-operatively.

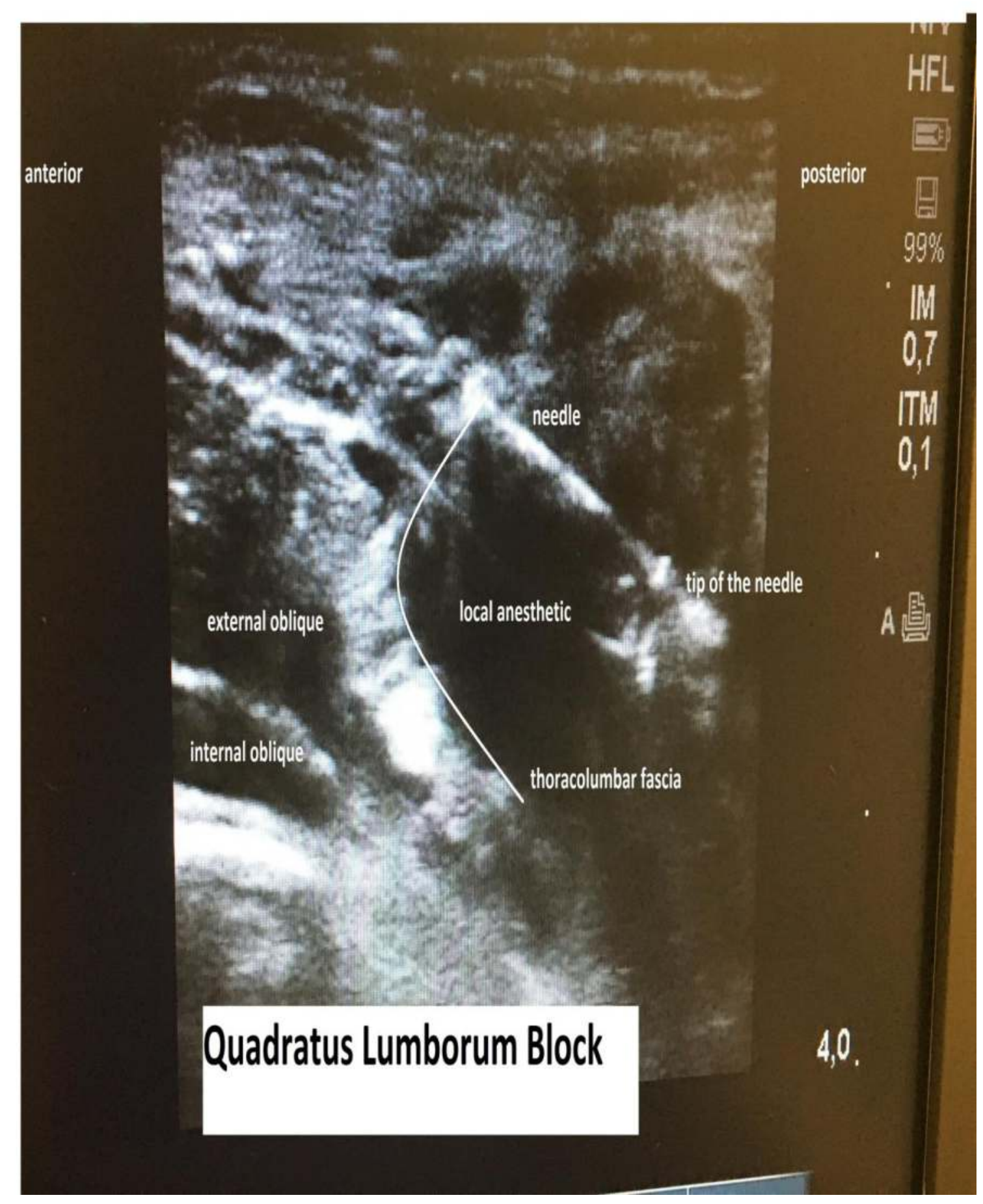

\section{DISCUSSION}

Pain control after laparotomy with intravenous analgesia is difficult without the use of large doses of opioids. Effective pain management is of great importance in a puerperal patient with the need for breastfeeding of the newborn. The possibility of bacteremia is a relative contraindication for epidural catheterization. In this case report, the bilateral single-shot QLB has been shown to be effective in pain control without the need for IV opioids or epidural catheterization. 